Felix Wachter, Adrian P. Regensburger, Antonia Sophia Peter, Ferdinand Knieling, Alexandra L. Wagner, David Simon, André Hoerning, Joachim Woelfle, Klaus Überla, Antje Neubert and Manfred Rauh*

\title{
Continuous monitoring of SARS-CoV-2 seroprevalence in children using residual blood samples from routine clinical chemistry
}

https://doi.org/10.1515/cclm-2022-0037

Received January 14, 2022; accepted February 14, 2022;

published online February 25, 2022

\section{Abstract}

Objectives: The assessment of SARS-CoV-2 infections in children is still challenging, but essential for appropriate political decisions. The aim of this study was to investigate whether residual blood samples can be used for SARS-CoV2 seroprevalence monitoring in pediatrics.

Methods: In this repeated cross-sectional cohort study, anonymous residual blood samples from pediatric patients aged 0-17 years were collected in three time-periods (Oct.Nov. 2020, April 2021, and June-July 2021) and analyzed for SARS-CoV-2 Spike protein (anti-S) and nucleocapsid (antiN) antibodies using commercial antibody assays. 28 reactive samples were used to compare antibody levels with a pseudotyped neutralization assay. The results were further compared to the official national COVID-19 surveillance data to calculate the number of unreported cases.

Felix Wachter and Adrian P. Regensburger contributed equally to this work.

Antje Neubert and Manfred Rauh contributed equally to this work.

*Corresponding author: Manfred Rauh, Department of Pediatrics and Adolescent Medicine, Friedrich-Alexander-University (FAU) ErlangenNürnberg, Loschgestr. 15, 91054 Erlangen, Germany, Phone: +49 9131 85 33118, E-mail: manfred.rauh@uk-erlangen.de

Felix Wachter, Adrian P. Regensburger, Ferdinand Knieling, Alexandra L. Wagner, André Hoerning, Joachim Woelfle and Antje Neubert, Department of Pediatrics and Adolescent Medicine, Friedrich-Alexander-University (FAU) Erlangen-Nürnberg, Erlangen, Germany, E-mail: felix.wachter@web.de (F. Wachter). https://orcid.org/0000-0003-1010-050X (F. Wachter) Antonia Sophia Peter and Klaus Überla, Institute of Clinical and Molecular Virology, Friedrich-Alexander University (FAU) ErlangenNürnberg, Erlangen, Germany

David Simon, Department of Internal Medicine 3, Friedrich-Alexander University (FAU) Erlangen-Nürnberg, Erlangen, Germany
Results: In total, $\mathrm{n}=2,626$ individual blood samples were analyzed. In this unvaccinated pediatric cohort anti-S and anti-N antibody seroprevalence increased over the three time periods (anti-S: $1.38-9.16 \%$, and $14.59 \%$; anti-N: $1.26 \%$, to $6.19 \%$, and $8.56 \%$ ). Compared to the national surveillance data this leads to a 3.93-5.66-fold increase in the number of unreported cases. However, a correlation between the cumulative incidence of the individual provinces and our assigned data was found ( $\mathrm{r}=0.74, \mathrm{p}=0.0151)$. In addition, reactive samples with anti-S and anti-N and samples with only anti-S showed neutralization capabilities (11/14 and $8 / 14$, respectively). Anti-S levels were not significantly different between age groups and sexes (all $\mathrm{p}>0.05$ ).

Conclusions: The present study suggests that residual blood samples from routine laboratory chemistry could be included in the estimation of the total SARS-CoV-2 seroprevalence in children.

Keywords: COVID-19; pediatrics; prevalence; SARS-CoV-2.

\section{Introduction}

Almost two years after the emerge of the novel Severe Acute Respiratory Syndrome Corona Virus (SARS-CoV-2) in 2019, the coronavirus disease 2019 (COVID-19) pandemic is still challenging people and health care systems around the world. There have been multiple studies characterizing basic virology, receptor use and differences to other Coronaviruses as well as epidemiological and clinical characteristics of the virus and health outcome $[1,2]$. However, the role of the virus in children remains controversially discussed and stills lacks investigations. So far, children seem to experience mild and often asymptomatic courses of COVID-19 [3-5]. Severe complications such as the multisystem inflammatory syndrome are extremely rare [3, 4]. But, since children are known to be responsible for spreading viral respiratory infections in general [6], their role in the SARS-CoV-2 pandemic has been extensively discussed. The reported incidence for children in Germany was still low in early June 2021 (3.5\%) with a 
steady increase up to $10.2 \%$ in December 2021 (https:// survstat.rki.de from 02.01.2022). However, the number might still be underestimating the real extent of infections in the pediatric population, as a lot of infections remain asymptomatic [3-5, 7], potentially leading to a high number of unreported infections. The surveillance of the infectious spread, vaccination status and state of herd immunity this difficult to reach part of the population is essential for political decision making and medical guidelines for children.

The use of commercially available nucleocapsid and spike protein assays for antibody detection showed accurate results in detecting previous SARS-CoV-2 infections in symptomatic adults [8] and even asymptomatic children [5]. Instead of incidence monitoring by positive PCR tests, focusing on antibody prevalence monitoring might allow a more accurate description of the dimension of rising infections [9] and vaccinations, especially in the youngest and asymptomatic population [5]. Seroprevalence studies have been performed in various regions and populations, but data regarding children remain scarce and vary widely among geographic regions [10]. The latter studies are hampered by the difficulty to perform studies in children due to various ethical regulations, the required large sample size and additional blood samples from the children. Therefore, residual blood samples from routine laboratory diagnostics could serve as an easily accessible source for studying the performance of different antibody assays for SARS-CoV-2 and also for real-time monitoring of the total SARS-CoV-2 seroprevalence [11]. Herein we present this approach to study the SARS-CoV-2 prevalence in a pediatric cohort at a large tertiary pediatric health care provider in Germany during the COVID-19 pandemic.

\section{Materials and methods}

\section{Study design}

This repeated cross-sectional cohort study was conducted during three time periods between (1) October 16th and November 16th, 2020, (2) April 1st and April 31st, 2021, and (3) June 15th and July 15th, 2021 at the Department of Pediatrics and Adolescent Medicine of the University Hospital Erlangen. Taking into account that seroconversion takes 7-14 days [12], time periods were approximately before the second, between second and third, and after the third wave of infections in Germany (please see Figure 1). Residual blood samples (lithium heparin plasma) of all inpatients and outpatients aged $0-17$ years with a visit during the time periods and a routine diagnostic blood test in the laboratory of the Hospital were stored for possible inclusion for the SARS-CoV-2 antibody analyses. From patients with numerous blood samples only one sample was used for the analyses. Samples with not enough residual blood were excluded.

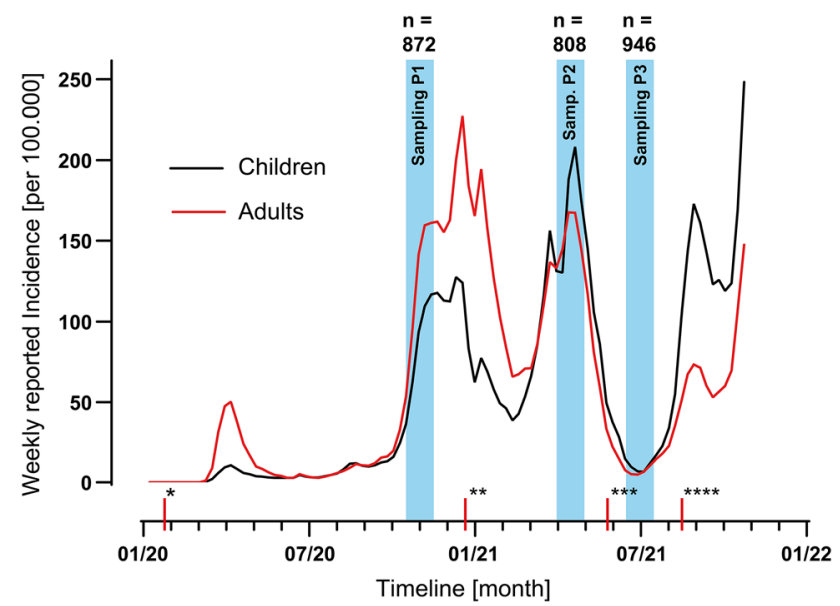

Figure 1: Weakly number of COVID-19 infections in Germany reported by the national authorities (Robert-Koch-Institute). Data plots were derived from https://survstat.rki.de (accessed November 6, 2021). Sample collection periods are highlighted blue with respective number of included children. Sampling 1: October 16th to November 16th,2020, sampling 2: April 1st to April 31st, sampling 3: June 15th to July 15th, 2021 *January 27, 2020 first case of COVID-19 in Germany. ${ }^{*}$ December 21, 2020 mRNA vaccine approval by the European Medicines Agency (EMA) for adults. *** May 28, 2021 mRNA vaccine approval by EMA for children aged

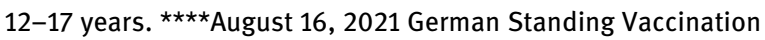
Committee (STIKO) recommendation for vaccination for all children 12-17 years.

The primary outcome measures of the study were the SARS-CoV-2 seroprevalence at the respective time points. Secondary outcome measures were quantitative antibodies against SARS-CoV-2, the rate of SARS-CoV-2 antibodies in comparison between time periods and stratification with patient characteristics (age and gender).

The study was approved by the Ethics Committee of the FriedrichAlexander University Erlangen-Nürnberg (Nr. 405_20 Bc), registered at clinicaltrials.gov (NCT04581148) and performed in adherence with the Helsinki Declaration (as revised in 2013). Consent was not required, as all analyses were performed anonymously.

\section{Test procedures}

All blood samples (lithium heparin plasma) were analyzed with the Roche Elecsys ${ }^{\circledR}$ Anti-Sars-CoV-2 (anti-N) and the Roche Elecsys ${ }^{\circledR}$ AntiSars-CoV-2 S (anti-S) assay as described in below. 28 samples reactive for either both, anti-N and anti-S ( $n=14)$ or anti-S only $(n=14)$ in the Roche Elecsys ${ }^{\circledR}$ assay from patients $<12$ years of age, were randomly chosen for comparison with ELISA and Pseudo Neutralization assay as described below.

\section{Roche Elecsys ${ }^{\circledR}$ anti-SARS-CoV-2 assay and anti- SARS-CoV-2-S assay}

All eligible blood samples were centrifuged and stored as plasma at $-15{ }^{\circ} \mathrm{C}$. Analyses were performed in batches of $n=60$ samples at the central laboratory of the Department of Pediatrics and Adolescent Medicine, Friedrich-Alexander-University (FAU) Erlangen-Nürnberg. 
After thawing, plasma samples were centrifuged at 3,000 runs per minute for $5 \mathrm{~min}$. Roche $\mathrm{N}$ and Roche $\mathrm{S}$ antibody assay were carried out using the Cobas e 601 module according to the manufacturer's protocol. Samples with no detectable antibody levels were discarded, reactive samples were frozen again and stored at $-15{ }^{\circ} \mathrm{C}$ for neutralizing assays.

For qualitative testing we used the electrochemiluminescence immunoassay (ECLIA) Elecsys ${ }^{\circledR}$ Anti-SARS-Cov-2 assay detecting antibody titer of all antibody isotypes (IgM, IgA, IgG) [13], and Elecsys ${ }^{\circledR}$ Anti-SARS-CoV-2-S immunoassay (ACOV2S) for the in vitro quantitative determination of antibodies (including IgG) to the SARS-CoV-2 spike (S) protein. All samples were processed according to the manufacturer's instructions.

Test results exceeding $0.8 \mathrm{U} / \mathrm{mL}$ were considered reactive, following the manufacturer's suggested cut-of-index. Values between 0.40 and $250 \mathrm{U} / \mathrm{mL}$ represent the linear range. Samples above $250 \mathrm{U} / \mathrm{mL}$ were automatically diluted into the linear range of the assay (realized dilutions in this study: 1:10 or 1:100) with Diluent Universal (Roche Diagnostics, Mannheim, Germany). The analyzer automatically multiplies diluted results with the dilution factor, which in the applied setting enabled an upper limit of quantification of $25,000 \mathrm{U} / \mathrm{mL}$ for these analyses. Four samples were not diluted beyond $250 \mathrm{U} / \mathrm{mL}$ or $2500 \mathrm{U} / \mathrm{mL}$. The assigned $\mathrm{U} / \mathrm{mL}$ are equivalent to Binding Antibody Units (BAU)/mL as defined by the first World Health Organization (WHO) International Standard for antiSARS-CoV-2 immunoglobulin (NIBSC code 20/136).

Samples with spike antibodies above $1000 \mathrm{U} / \mathrm{mL}$ and no nucleocapsid antibodies $(<0.12 \mathrm{~S} / \mathrm{Co})$ were excluded from further analyses as we assumed that these patients had been vaccinated as nativelyinfected individuals developed a lower and more heterogeneous antibody response to SARS-CoV-2 [14].

\section{Nucleocapsid and spike ELISA}

Binding of antibodies to the nucleocapsid or spike protein was assessed with a commercially available ELISA based assay. To this end an NCP or S1 ELISA kit (both Euroimmun, Lübeck, Germany; EI 2606-9601 G, EI 2606-9601 G, respectively) was employed. The microtiter plates were treated as instructed by the manufacturer. Following the plates were acquired on a multilabel microplate reader VICTOR X5 (PerkinElmer, Waltham, USA). The cut off for positive samples was set and data analysis was performed as recommended by the manufacturer.

\section{Pseudovirus neutralization assay}

Neutralization assays were performed as described previously $[15,16]$. Briefly, lentiviral particles pseudotyped with the D614G spike mutant of SARS-CoV-2 were incubated for $1 \mathrm{~h}$ at $37^{\circ} \mathrm{C}$ with serially diluted patient sera, ranging from 1:20 to 1:2,500, in DMEM (Thermo Fisher scientific, Walham, USA) supplemented with 10\% Fetal Calf Serum (Capricorn, Ebsdorfergrund, Germany), $5 \mathrm{~mL}$ Glutamax (Thermo Fisher scientific, Waltham, USA) and 100 units/mL penicillin/streptomycin (Sigma-Aldrich, St. Louis, USA) [17]. Following the mixture was added onto $2 \times 10^{4}$ HEK293T ACE2 overexpressing cells, seeded per well of a 96-well flat bottom plate (Greiner, Kremsmünster, Austria) on the day prior to the experiment [18]. $48 \mathrm{~h}$ after infection the cells were washed twice with PBS and $50 \mu \mathrm{l}$ of PBS and $25 \mu \mathrm{L}$ of ONE-Glo ${ }^{\mathrm{TM}}$ (Promega, Madison, USA) were added per well. After $3 \mathrm{~min}$ the luciferase signal was measured on a multilabel microplate reader VICTOR X5 (PerkinElmer, Waltham, USA). The reciprocal serum dilution IC50s were determined with Prism 9-GraphPad (San Diego, CA, USA). In case sera that did not reach neutralization by at least $50 \%$ the IC50s were set to the lowest reciprocal serum dilution of 20 .

\section{Clinical and epidemiological data}

The anonymized data was analyzed for sex, age and zip code. We categorized participants in the following age groups. Newborns: 031 days old. Infants: 32 days-2 years old. Preschool: 3-5 years old. Elementary school: 6-9 years old. Secondary school: 10-14 years old. Adolescents: $15-17$ years old. Further specific clinical data were not available due to the anonymization procedure. As large tertiary pediatric health care provider a broad variety of acute and chronic diseases in children are treated. To be able to account for vaccinated adolescents, we categorized children older than 12 with no nucleocapsid response $(<0.12 \mathrm{~S} / \mathrm{Co})$ and a spike level above $1000 \mathrm{U} / \mathrm{mL}$ as presumably vaccinated. Supported by data from adults [19], this assumption was made after analyzing the anti-S levels of 82 participants of our study with no anti-N response. Herein two separate clusters of immune response were found. One with anti-S levels between $1 \mathrm{U} / \mathrm{mL}$ and $500 \mathrm{U} / \mathrm{mL}$ and one with anti-S levels $\geq 1.200 \mathrm{U} / \mathrm{mL}$, but none in between. Hereafter, we have set the cut off at $1000 \mathrm{U} / \mathrm{mL}$.

Furthermore, children older than 12 with a spike antibody level above $10,000 \mathrm{U} / \mathrm{mL}$ and a nucleocapsid response were classified as presumably vaccinated and previously infected according to the assumption that the immune response in these patients is multiplied [20-22].

Only unvaccinated children were used for seroprevalence estimates, calculation of the number of unreported cases and antibody level analyses.

Furthermore, the Public Health Institute of Germany (RobertKoch-Institute) reported the number of PCR-confirmed Covid-19 cases in the Bavarian districts and the corresponding age and sex of each positive tested person (https://survstat.rki.de, accessed November 14, 2021). For further comparisons, we assigned each patient of our study the respective cumulative incidence corresponding to its province of residency, age and sex. Newborns and participants without patient data were excluded from the calculation of unreported cases. When determining the cumulative incidence, we defined the cutoff date to be the day 8-11 days prior to the time period of sampling, ergo October 4, 2020, March 21, 2021 and June 6, 2021, assuming a 7-14 days time interval for seroconversion [12].

\section{Analytic methods and statistics}

Sex and age were characterized using descriptive statistics (number and percentage for sex, median and IQR for age). Seroprevalence of anti-S/anti-N during each time period was calculated as the number of patients with anti-S/anti-N reactive samples divided by the total number of included samples. For further analyses the anti-S seroprevalence was used. The number of unreported cases was calculated by dividing anti-S seroprevalence by the average representative cumulative incidence reported by the authorities. Seroprevalence rates were compared using Fisher's exact test. Data was analyzed for normal distribution by Shapiro-Wilk test prior to further analyses. Correlations are given by either Pearson (r) or Spearman $\left(\mathrm{r}_{\mathrm{s}}\right)$ correlation coefficient, as appropriate. Anti-S levels between age groups were 
compared by Kruskal-Wallis test with Dunn's multiple comparison test. Anti-S levels between sexes was compared using Mann-Whitney test. A p-value $<0.05$ was regarded as statistically significant.

\section{Results}

\section{Participants}

A total of 2,626 individual blood samples were included in the study. 872 during the first (Oct.-Nov. 2020), 808 during the second (April 2020) and 946 during the third time period (June-July 2021), respectively. Median age was 8.7 years (IQR 3.4-13.6) in the first, 8.4 years (IQR 3.2-14.0) in the second and 8.6 years (IQR 3.8-13.8) in the third time period. $47.4 \%$ of patients in the first, $45.8 \%$ in the second and $45.9 \%$ in the third time period were female (Figure 1 and Table 1). In total, 224 samples were reactive in anti-S, 12 samples in time period one, 74 in time period two and 138 in time period three. 142 samples were reactive in anti-N, 11 samples in time period one, 50 in time period two and 81 in time period three (Supplemental Table 1).

mRNA vaccines were not approved by the European Medicines Agency (EMA) for children during time period 1 and 2. During time period 3, a mRNA vaccine was approved for children aged 12-17 years. However, vaccination was not recommended by the German Standing Vaccination Committee (STIKO) which is essential for pediatricians for the broad application of vaccines. Furthermore, as reported by national data, only $1.2 \%$ of Bavarian citizens aged younger than 18 years were vaccinated by the start of the third sampling period (https:/github.com/evilpie/ Impfquotenmonitoring/blob/master/Impfquotenmonitoring2021-06-16T07:58:08.xlsx, retrieved 02.02.2022). Therefore the number of vaccinated children was considered low.

\section{Seroprevalence}

Seroprevalence for the SARS-CoV-2 nucleocapsid protein antibodies increased from 1.26 to $6.19 \%(\mathrm{p}=0.00001)$ and
$8.56 \%$ ( $\mathrm{p}=0.0682)$ during the sample periods, while seroprevalence for the spike protein increased from 1.38 to 9.16\% $(\mathrm{p}=0.00001)$ and $14.59 \%(\mathrm{p}=0.0005)$. In the third time period (June 2021) $2.64 \%$ of the children showed anti-S antibodies $>1000 \mathrm{U} / \mathrm{mL}(\mathrm{n}=25)$, but no anti- $\mathrm{N}$ immune response and were therefore classified as presumably vaccinated (Figure 2A). Spike protein antibody prevalence was particularly high in the adolescent population during the third time period. Nucleocapsid antibody prevalence in this age group was similar to secondary school children (Figure 2B and Supplemental Table 1).

While in the second time period, prevalence of anti-S was similar between male and female participants ( $p>0.99$ ), in the first and third time period anti-S seroprevalence was lower in female participants (1st: $p=0.15$ and 3rd: $p=0.0161$ ) (Figure 2C and Supplemental Table 1).

The five provinces with the highest number of participants in time period 2 and 3 were analyzed for their respective reported cumulative incidence and the obtained seroprevalence. While progression of the seroprevalence within the districts was different, correlation between incidence and prevalence was high $(r=0.74, p=0.0151)$ (Figure 2E).

\section{Quantitative and qualitative antibody analyses}

Of the 225 participants classified seropositive, 141 (62.7\%) showed both anti-N and anti-S antibodies, 83 (36.9\%) were only reactive in the anti-S assay and $1(0.4 \%)$ was only reactive in anti-N assay. This yields at a total concordance of $62.7 \%$ and a discordance of $37.3 \%$. However, nucleocapsid protein antibody response correlated with spike protein antibody levels $(r=0.65, p<0.0001)$ (Figure 3A).

We then further analyzed a subset of $n=28$ reactive samples for anti-S and anti-N verification and their respective neutralization capacity. To exclude vaccinated patients, samples from children younger than 12 years were chosen. All samples were analyzed by ELISA for $\mathrm{N}$ and $\mathrm{S}$

Table 1: Patient characteristics.

\begin{tabular}{lrrr}
\hline Sample period & 1 (Oct. 15 - Nov. 15, 2020) & 2 (April 1 - April 31, 2021) & 3 (June 15 - July 15, 2021) \\
\hline Number of included samples, $n$ & 872 & 808 & 946 \\
Female, $n$ (\%) & $413(47.4 \%)$ & $370(45.8 \%)$ & $434(45.9 \%)$ \\
Age, days, mean (SD) & $3,130(2045)$ & $3,137(2,128)$ & $3,166(2064)$ \\
Age, years, mean (SD) & $8.6(5.6)$ & $8.6(5.8)$ & $8.7(5.7)$ \\
\hline
\end{tabular}




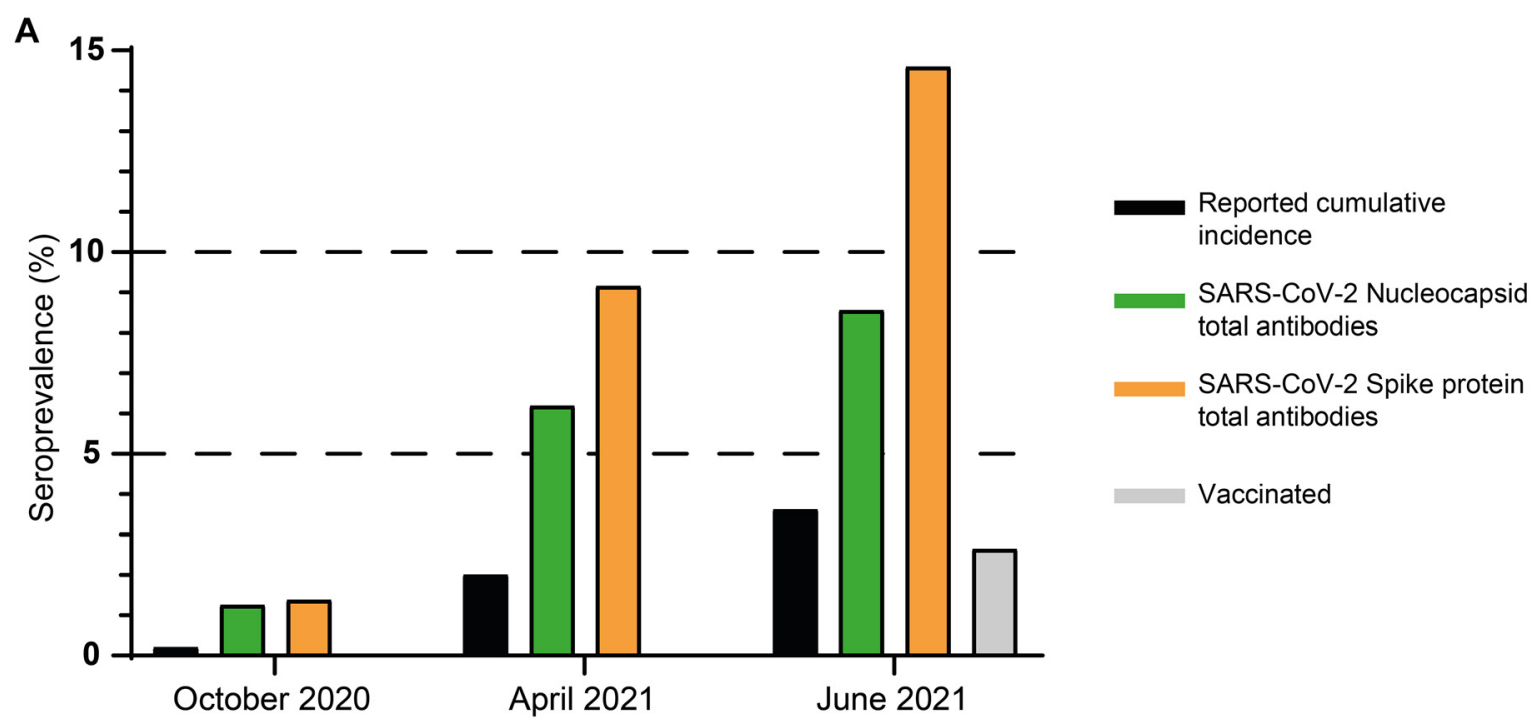

B

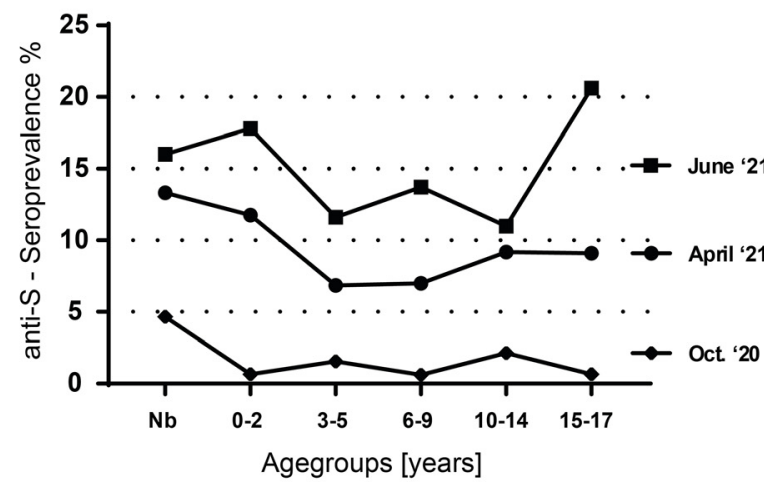

D

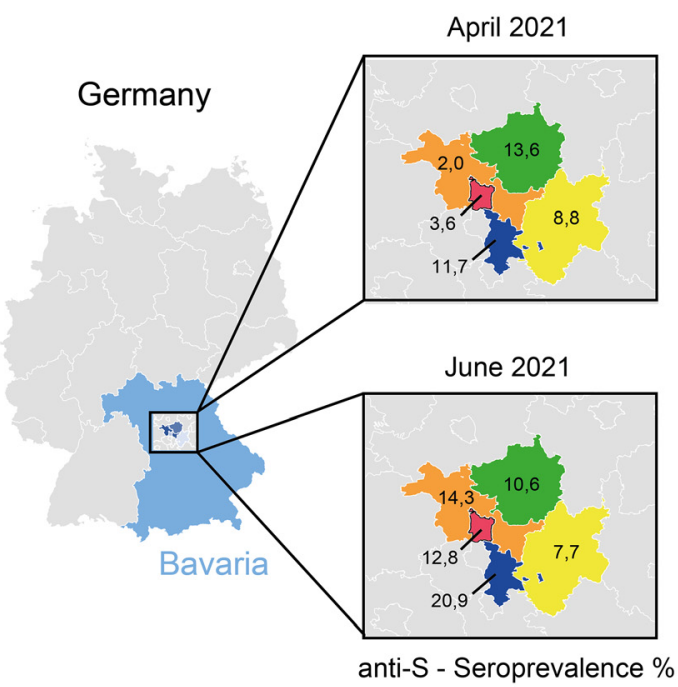

C

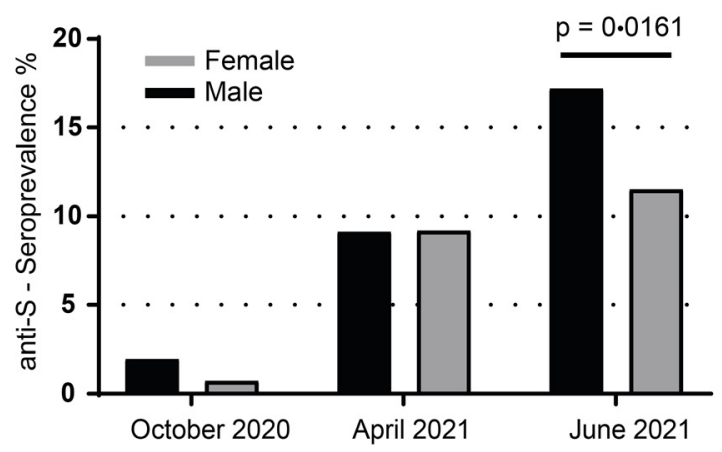

E

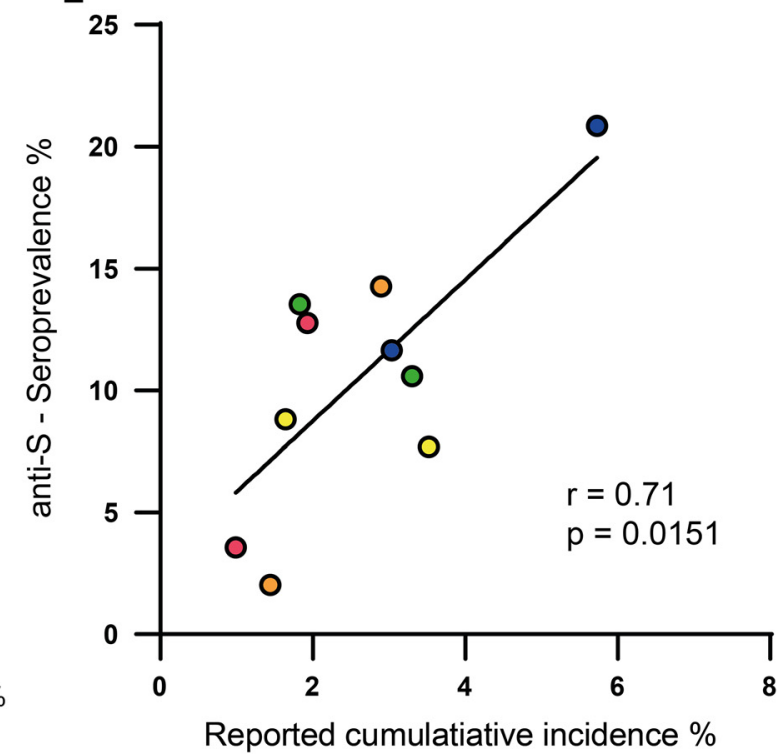

Figure 2: SARS-CoV-2 nucleocapsid and spike protein antibody seroprevalence during the time periods.

(A) Comparison between reported SARS-CoV-2 incidence by the Robert-Koch-Institute (https://survstat.rki.de, accessed 12.11.2021) and measured SARS-Cov-2 nucleocapsid and spike protein total antibody seroprevalence. (B) anti-S=SARS-Cov-2 spike protein total antibodies. Measured anti-S prevalence in different age groups during sampling periods 1-3. (C) anti-S=SARS-Cov-2 spike protein total antibodies. 
Measured anti-S prevalence between male and female participants. (D) anti-S=SARS-Cov- 2 spike protein total antibodies. Displayed are the five provinces with highest number of blood samples from patients admitted to the supraregional healthcare provider in this study (orange $0=$ LK Erlangen Hoechstadt, green=LK Forchheim, red=LK Erlangen, yellow=LL Nuremberger Land, blue=LK Nuremberg) with the anti-S prevalences during samping period 2 (April 21) and sampling period 3 (June 2021). Sampling period 1 was excluded due to the limited number of positive cases $(n=5)$. (E) anti-S=SARS-Cov-2 spike protein total antibodies. Correlation of anti-S seroprevalence and reported cumulatative incidence for the five provinces with highest number of blood samples from patients admitted to the supraregional healthcare provider in this study (orange $\mathrm{O}=\mathrm{LK}$ Erlangen Hoechstadt, green=LK Forchheim, red=LK Erlangen, yellow $=$ LL Nuremberger Land, blue=LK Nuremberg) with the anti-S prevalences during samping period 2 (April 21) and sampling period 3 (June 2021). Sampling period 1 was excluded due to the limited number of positive cases $(n=5)$.

antibodies and a pseudotyped neutralization assay in two groups. Group one consisted of 14 samples that were reactive in anti-S only and group two of 14 samples reactive in anti-S and anti-N ECLIA (Roche Elecsys ${ }^{\circledR}$ ). The ELISA assay confirmed ECLIA results in most cases but remained unreactive in few samples (Supplemental Table 2). In group
A

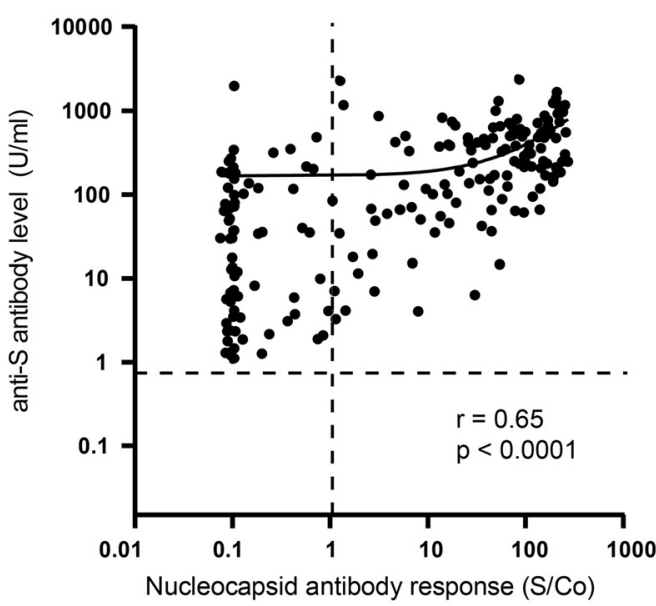

C

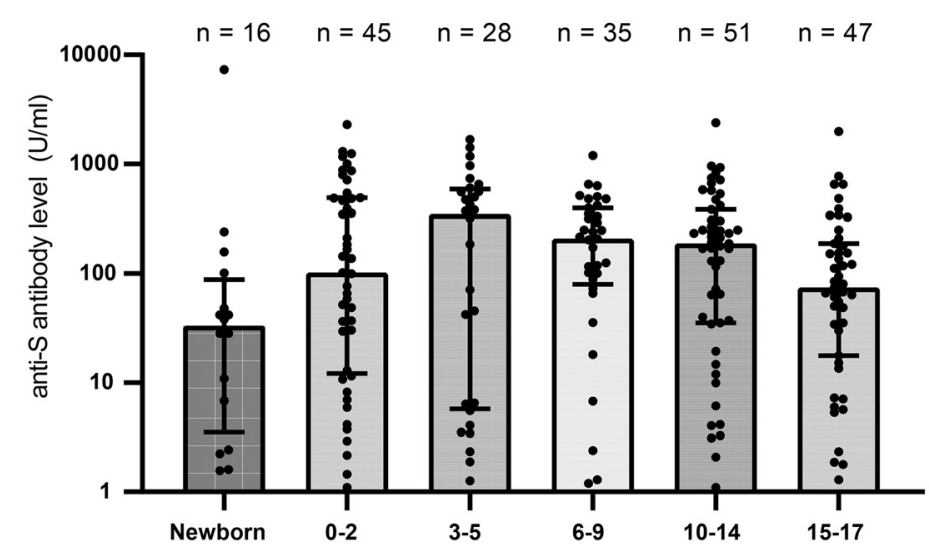

B Samples with neutralizing capacity

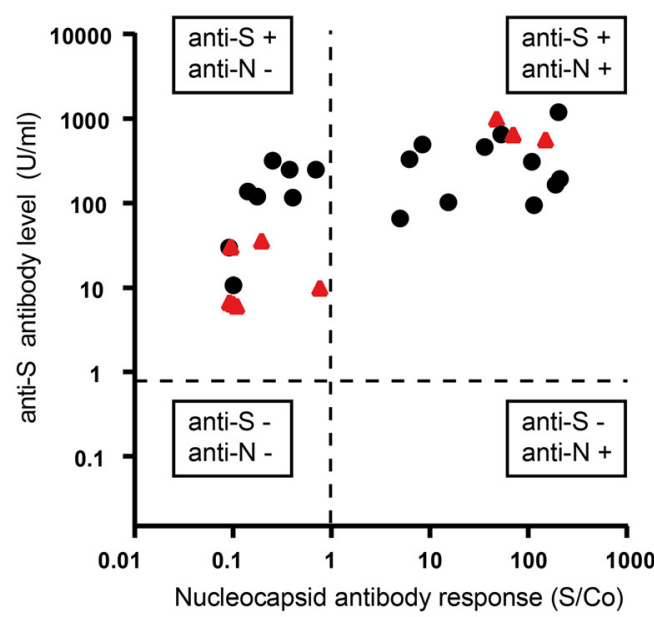

D

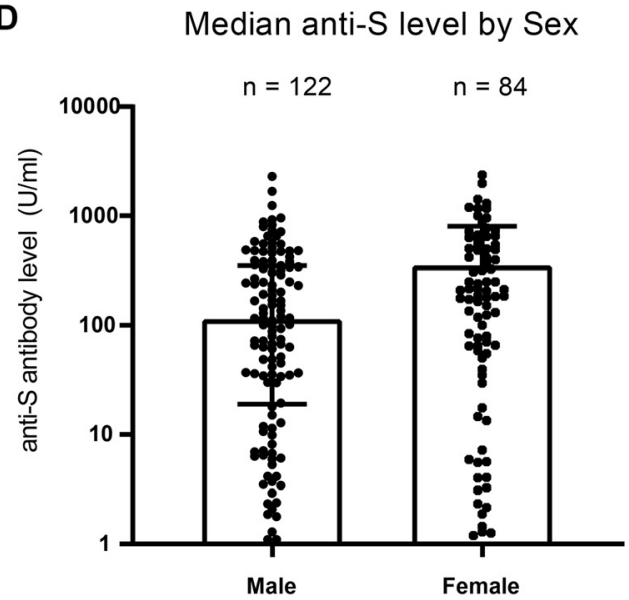

Figure 3: Quantitative and qualitative anti-S and anti-N analyses. anti-S=SARS-Cov-2 spike protein total antibodies.

(A) SARS-Cov-2 nucleocapsid antibody response was correlated with spike protein antibody levels of all reactive samples. Cut-off-indices for both assays are shown by the dotted lines. $r=$ Pearson correlation coefficient. $p \leq 0.05$ was regarded statistically significant. (B) Results of the neutralization assay. Red triangle=sample had no neutralizing capabilities. Black dot=sample had neutralizing capabilities. Cut-off-indices for both assays are shown by the dotted lines. $r=$ Pearson correlation coefficient. $p \leq 0.05$ was regarded statistically significant. (C) anti$\mathrm{S}=$ SARS-Cov- 2 spike protein total antibodies. anti-S levels in different age groups. Bars indicate median and IQR. $p \leq 0.05$ was regarded statistically significant. (D) anti-S=SARS-Cov-2 spike protein total antibodies. anti-S levels compared between male and female participants. Bars indicate median and IQR. $p \leq 0.05$ was regarded statistically significant. 
one (anti-S reactive only), neutralizing antibodies were found in 8 of 14, in group two (anti-S and anti-N reactive) in 11 of 14 samples (Figure 3B).

Median spike antibody level during the time periods varied from $151.9 \mathrm{U} / \mathrm{mL}$ (95\% Confidence interval [CI] 6.96 to 472.6 ) to $124.3 \mathrm{U} / \mathrm{mL}$ (CI 55.02 to 232.1 ) and $152.6 \mathrm{U} / \mathrm{mL}$ (CI 93.89 to 212.6). Median spike antibody level by age groups ranged from median $33 \mathrm{U} / \mathrm{mL}$ ( $\mathrm{CI} 2.43$ to 101.0 ) in newborns to median $346.1 \mathrm{U} / \mathrm{mL}$ (CI 6.5 to 553.3 ) in 3-5 years old children (all $\mathrm{p}>0.05$ ) (Figure 3C).

Furthermore, anti-S levels were similar between girls and boys (median 184.8, CI 118.8 to 250.0 vs. 113.5 , CI 67.55 to $170.0, \mathrm{p}=0.115$ ) (Figure 3D).

\section{Number of undetected cases}

For calculation of undetected cases, only participants above 31 days of age, to avoid cases of maternally transmitted antibodies, with anti-S reactive samples were included. Therefore, $n=829$ patients were analysed in the first time period. Herein, the anti-S seroprevalence was $1.21 \%$ and the reported cumulative incidence $0.21 \%$. This leads to a ratio of 5.66 unreported cases. In the second time period $n=761$ patients with a spike protein antibody seroprevalence of $8.94 \%$ were compared to the reported $\mathrm{cu}$ mulative incidence of $2.05 \%$, and in the third time period $\mathrm{n}=895$ participants with a spike protein antibody seroprevalence of $14.53 \%$ and a reported cumulative incidence of $3.70 \%$ lead to a ratio of unreported cases of 4.35 and 3.93, respectively (Figure 4A).

The number of unreported cases was higher in male patients in the first and third and similar in the second time period (Figure 4B). The ratio of unreported cases compared between age groups diverged between time periods. In time period one, with a limited number on $n=12$ cases, the highest ratio of unknown cases was found in 3-5 years old children. In time period 2 and 3, the highest number of unknown cases was highest in the youngest age group $(0-2$ years) (Figure 4C).

\section{Discussion}

In this study, a novel approach that was only using residual plasma from assigned patients to a large tertiary pediatric health care provider, showed a simple opportunity to monitor SARS-CoV-2 seroprevalence in children during the pandemic. From October 2020 to July 2021 a rising spike protein and nucleocapsid antibody seroprevalence was found in this cohort with a high number of unreported cases compared to official national surveillance data.

Although the seroprevalence naturally varies between geographic locations [10], a comparison with other pediatric seroprevalence studies in Germany revealed a good concordance. During the course of our study the anti-S seroprevalence increased continuously from 1.38 to $9.16 \%$, and $14.59 \%$. Similar numbers were found in Saxony and Berlin (0.5-2.0\%) during the first [23] and in Germany about the second (5.6-8.4\%) time period [24].

Interestingly, throughout all sampling periods, anti- $\mathrm{N}$ reactive samples were less frequently detected than anti-S reactive samples, what could not be attributed to vaccinations, as mRNA vaccines were not recommended by the national authority (STIKO) by the time of the study. Individual vaccinated adolescents $(n=25)$ during the third time period were excluded as presumably vaccinated by high anti-S (>1000 U/mL) and no anti-N antibodies. However, reduced immune response to vaccinations in patients with immunecompromise [25] could led to a small number of undetected cases of vaccinations during the third time period. To rule out a systematic error by using spike protein as indicator for a past SARS-CoV-2 infection, our findings were verified and confirmed by nucleocapsid and spike ELISA and a pseudotyped neutralization assay. Herein, neutralizing antibodies were found in only anti-S reactive samples, too. This phenomenon in children may partially be explained by the dependence of the immune response to the course of the disease [26] and goes in hand with the mild to asymptomatic SARS-CoV-2 infections in children [3, 4]. Furthermore, a distinct immune response to SARS-CoV2in children [27] with a preactivation and priming for virus sensing leads to early Interferon production with consecutive reduced virus replication [28] and weaker antibody response to the nucleocapsid, which is essential for virus replication [27, 29]. However, spike specific T-cell response is 2-fold higher in children and could be found in seronegative children, too [30]. In addition, half-life of anti-N seems to be shorter compared to the Receptor-bindingdomain with one study reporting that $50 \%$ of anti-N positive cases become seronegative after 195 days [31]. The concordance between anti-S and anti-N was previously described as $83.9 \%$ [32]. Our data suggests an even lower concordance of only $62.7 \%$, potentially influenced by a long period of time to the previous infection. So far, data focusing on the accuracy of the used antibody assays are based on adults [33] and mostly include hospitalized patients with severe, or at least symptomatic SARS-CoV-2 infections [8]. Currently, different assays are used in everyday pediatric clinical practice to detect past infections and monitor immune response after vaccination or are 
A

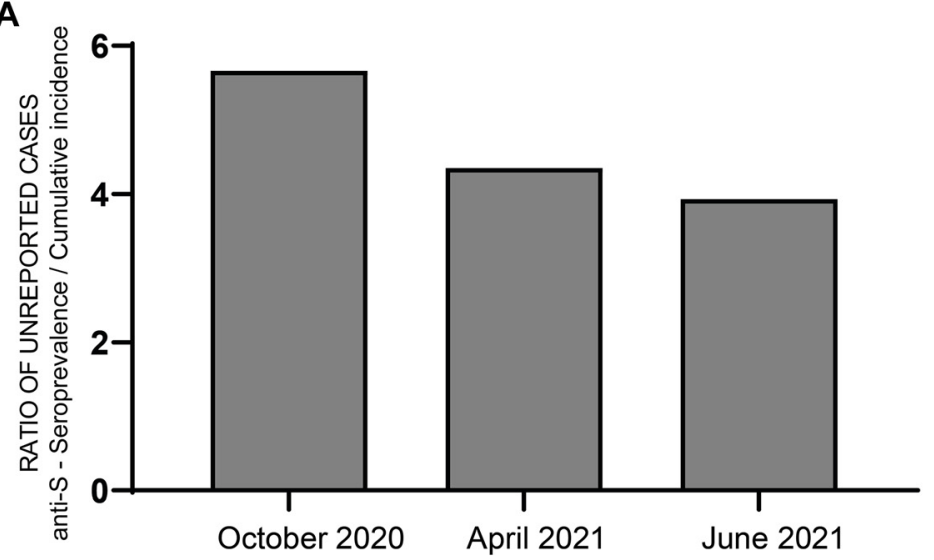

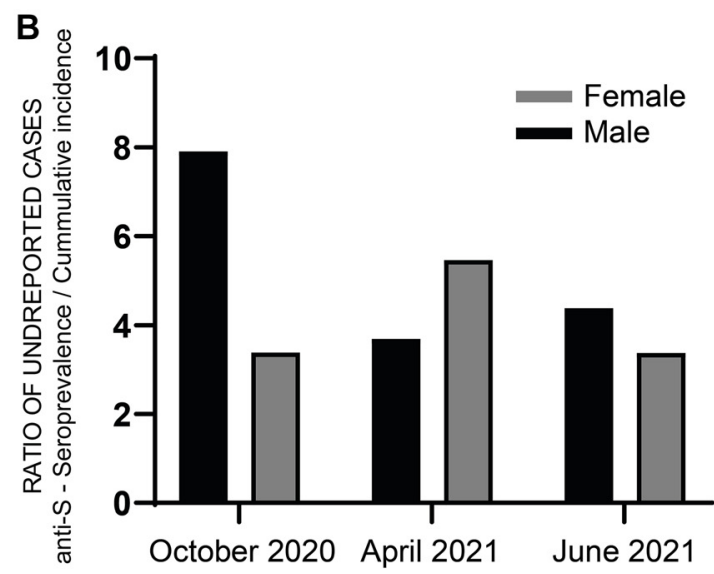

C

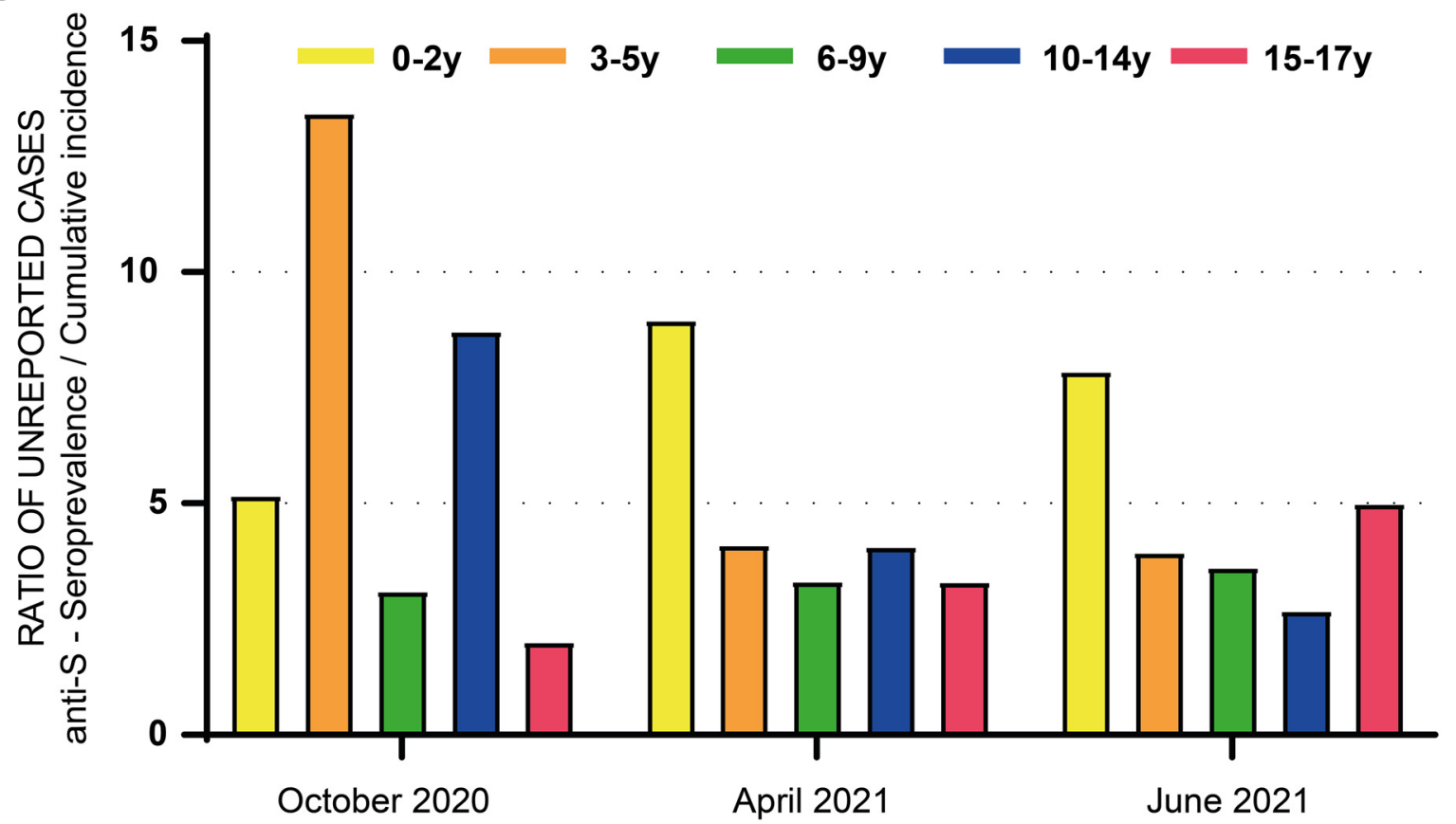

Figure 4: Number of unreported SARS-CoV-2 cases during the time periods. anti-S=SARS-Cov-2 spike protein total antibodies, $y=y e a r s$. (A) Ratio of unreported cases during the sample periods. Sampling 1: October 16th to November 16th, 2020, sampling 2: April 1st to April 31st, sampling 3: June 15th to July 15th, 2021. (B) Ratio of unreported cases during the sample periods. Sampling 1: October 16th to November 16th, 2020, sampling 2: April 1st to April 31st, sampling 3: June 15th to July 15th, 2021 divided by sex. (C) Ratio of unreported cases during the sample periods. Sampling 1: October 16th to November 16th, 2020, sampling 2: April 1st to April 31st, sampling 3: June 15th to July 15 th, 2021 divided by different age groups.

applied for multisystem-inflammatory-syndrome cases in children (MIS-C) and for work-up purposes in Long-Covid. Our data suggests that existing seroprevalence studies based on anti-N assays could underestimate the real extent of infections in unvaccinated pediatric cohorts. As shown, when comparing national surveillance data with our anti-S seroprevalence an up to 5.66-fold higher number of SARS-CoV 2 positive cases were found in our population.
The decrease of unknown cases during the time periods could be attributed to the political guidelines, including increased test strategies for children during the pandemic. After our first study time period in October 2020 systematic testing strategies in schools in Germany for the winter season 2021 were established. Our findings are in line with reported 6-fold higher number of unreported cases in Germany [34] after the first wave of infections, decreasing 
to 3-4-fold in February 2021 [24]. In contrast, unknown cases in adults were only 2 -fold higher, as reported by the Robert-Koch-Institute [35]. To analyze local differences of infection rates, the local reported cumulative incidence of infections was correlated with the prevalence of our patient cohort with their respective zip codes, revealing a good correlation indicating a potential transferability of the data from our hospital to the respective provinces. However, the approach to compare cumulative incidences from positive PCR tests with the seroprevalence through a ratio might influence the detected number of unknown cases and should be considered thoughtfully.

In contrast to our approach, which requires only material costs, as all other steps can be implement in routine clinical processing of blood samples without additional personnel, field studies for seroprevalence monitoring require the provision of additional medical staff, extensive organization, high logistic effort for random sampling, and rely on participation, possibly comprising a participation bias [32]. The main limitation of our study represents the missing medical history regarding vaccination status and SARS-CoV-2 infections. However, due to the large cohort and the analysis within three time periods without recommended vaccination of children the influence to the present study seems to be limited. Known from adults, that distinct SARS-CoV-2 antibody levels are higher in vaccinated compared to naturally infected persons [19], estimated cut offs were chosen to allocate anti-S positive only samples to vaccinated individuals. The number of $2.64 \%$ vaccinated children derived from the used cut offs is in line with the official number of vaccinated children (1.2\%) at the beginning of the third time period. However, the relatively high anti-S antibody levels in adolescents during the third time period might also indicate a certain portion of false classified samples. As we cannot resolve this with our study design, further studies are needed to provide details about the antibody levels in (un-) vaccinated children and the influence of breakthroughs and hybrid immunity and in comparison to adults [22].

Another limitation of our study is the unknown number of immunodeficient patients. This could influence the transferability of our data to the overall population of healthy children. A general limitation in assessing seroprevalence by anti-S arises from the fact that no distinction can be made between vaccinated and previously infected individuals, a fact that is becoming increasingly apparent as vaccination rates in children increase. However, monitoring of the total seroprevalence is necessary to determine achievement of herd immunity, which might be more important than the differentiation between past SARS-CoV2 infection or vaccination. Especially in children below 5 years of age, where continuous surveillance is lacking the most, this approach could overcome the aforementioned difficulties [36]. With the new "Omicron" wave and new variants on the horizon, the surveillance of antibody status in pediatrics may be brought into focus even more.

We conclude that an estimations of the total seroprevalence can be easily implemented into daily laboratory routines using only routine residual blood samples, which are available from almost all pediatric patients.

Acknowledgments: We thank the Laboratory staff of the Department of Pediatrics and Adolescent Medicine and the Institute of Virology. The present work was performed in fulfillment of the requirements for obtaining the degree "Dr. med." for FW. The present work was performed in (partial) fulfillment of the requirements for obtaining the degree "Dr. rer. biol. hum." for APR.

Research funding: The project was funded by the Interdisciplinary Center for Clinical Research (IZKF) junior project (J089) and Clinician Scientist Program (CSP) at the University Hospital of the Friedrich-Alexander-Universität (FAU) Erlangen-Nürnberg to A.P.R. Further funding was provided by the Bavarian Staatsministerium für Wissenschaft und Kunst (TiKoCo) to K.Ü.

Author contribution: A.P.R., A.N., and M.R. conceived the idea of the study. Data collection was completed by F.W. and M.R. The first draft of the manuscript was written by F.W. and A.P.R. The data was analyzed by F.W., A.P.R., A.S.P., M.R., and A.N. Data was interpreted by F.W., A.P.R., A.S.P., F.K., A.L.W., D.S., A.H., J.W., K.Ü., A.N., and M.R. The manuscript was critically reviewed by all authors. All authors have accepted responsibility for the entire content of this manuscript and approved its submission.

Competing interests: Authors state no conflict of interest. Informed consent: Informed consent was not required, as all analyses were performed anonymously.

Ethical approval: The study was approved by the Ethics Committee of the Friedrich-Alexander University ErlangenNürnberg (Nr. 405_20 Bc) and performed in adherence with the Helsinki Declaration (as revised in 2013).

Data availability: Individual patient data is not available. The datasets analyzed during the current study are available from the corresponding author on reasonable request.

\section{References}

1. Hu B, Guo H, Zhou P, Shi ZL. Characteristics of SARS-CoV-2 and COVID-19. Nat Rev Microbiol 2021;19:141-54. 
2. Wu Z, McGoogan JM. Characteristics of and important lessons from the coronavirus disease 2019 (COVID-19) outbreak in China: summary of a report of 72314 cases from the Chinese center for disease control and prevention. JAMA 2020;323:1239-42.

3. Dong Y, Mo X, Hu Y, Qi X, Jiang F, Jiang Z, et al. Epidemiology of COVID-19 among children in China. Pediatrics 2020;145: e20200702.

4. Ludvigsson JF. Systematic review of COVID-19 in children shows milder cases and a better prognosis than adults. Acta Paediatr 2020;109:1088-95.

5. Renk H, Dulovic A, Seidel A, Becker M, Fabricius D, Zernickel M, et al. Robust and durable serological response following pediatric SARS-CoV-2 infection. Nat Commun 2022;13:128.

6. Park S, Lee Y, Michelow IC, Choe YJ. Global seasonality of human coronaviruses: a systematic review. Open Forum Infect Dis 2020;7: ofaa443.

7. Poletti P, Tirani M, Cereda D, Trentini F, Guzzetta G, Sabatino G, et al. Association of age with likelihood of developing symptoms and critical disease among close contacts exposed to patients with confirmed SARS-CoV-2 infection in Italy. JAMA Netw Open 2021;4: e211085.

8. Deeks JJ, Dinnes J, Takwoingi Y, Davenport C, Spijker R, TaylorPhillips S, et al. Antibody tests for identification of current and past infection with SARS-CoV-2. Cochrane Database Syst Rev 2020;6:CD013652.

9. Byambasuren O, Dobler CC, Bell K, Rojas DP, Clark J, McLaws ML, et al. Comparison of seroprevalence of SARS-CoV-2 infections with cumulative and imputed COVID-19 cases: systematic review. PLoS One 2021;16: e0248946.

10. Rostami A, Sepidarkish M, Leeflang MMG, Riahi SM, Nourollahpour Shiadeh M, Esfandyari S, et al. SARS-CoV-2 seroprevalence worldwide: a systematic review and metaanalysis. Clin Microbiol Infect 2021;27:331-40.

11. Kugeler KJ, Podewils LJ, Alden NB, Burket TL, Kawasaki B, Biggerstaff BJ, et al. Assessment of SARS-CoV-2 seroprevalence by community survey and residual specimens, Denver, Colorado. Publ Health Rep 2022;137:128-36.

12. Wolfel R, Corman VM, Guggemos W, Seilmaier M, Zange S, Muller $M A$, et al. Virological assessment of hospitalized patients with COVID-2019. Nature 2020;581:465-9.

13. Muench P, Jochum S, Wenderoth V, Ofenloch-Haehnle B, Hombach M, Strobl M, et al. Development and validation of the Elecsys anti-SARS-CoV-2 immunoassay as a highly specific tool for determining past exposure to SARS-CoV-2. J Clin Microbiol 2020;58:e01694-20.

14. Jochum S, Kirste I, Hortsch S, Grunert VP, Legault H, Eichenlaub U, et al. Clinical utility of Elecsys Anti-SARS-CoV-2 S assay in COVID19 vaccination: an exploratory analysis of the mRNA-1273 phase 1 trial. medRxiv 2021;12:798117.

15. Lapuente D, Fuchs J, Willar J, Vieira Antao A, Eberlein V, Uhlig N, et al. Protective mucosal immunity against SARS-CoV-2 after heterologous systemic prime-mucosal boost immunization. Nat Commun 2021;12:6871.

16. Dispinseri S, Secchi M, Pirillo MF, Tolazzi M, Borghi M, Brigatti C, et al. Neutralizing antibody responses to SARS-CoV-2 in symptomatic COVID-19 is persistent and critical for survival. Nat Commun 2021;12:2670.

17. Hoffmann M, Arora P, Gross R, Seidel A, Hornich BF, Hahn AS, et al. SARS-CoV-2 variants B.1.351 and P.1 escape from neutralizing antibodies. Cell 2021;184:2384-93.
18. Herrmann A, Jungnickl D, Cordsmeier A, Peter AS, Uberla K, Ensser A. Cloning of a passage-free SARS-CoV-2 genome and mutagenesis using red recombination. Int J Mol Sci 2021;22: 10188.

19. Assis R, Jain A, Nakajima R, Jasinskas A, Khan S, Palma A, et al. Distinct SARS-CoV-2 antibody reactivity patterns elicited by natural infection and mRNA vaccination. npj Vaccines 2021;6: 132.

20. Turner JS, O'Halloran JA, Kalaidina E, Kim W, Schmitz AJ, Zhou JQ, et al. SARS-CoV-2 mRNA vaccines induce persistent human germinal centre responses. Nature 2021;596:109-13.

21. Wang Z, Muecksch F, Schaefer-Babajew D, Finkin S, Viant C, Gaebler $C$, et al. Naturally enhanced neutralizing breadth against SARS-CoV-2 one year after infection. Nature 2021; 595:426-31.

22. Bates TA, McBride SK, Leier HC, Guzman G, Lyski ZL, Schoen D, et al. Vaccination before or after SARS-CoV-2 infection leads to robust humoral response and antibodies that effectively neutralize variants. Sci Immunol 2022;7:eabn8014.

23. Thamm R, Buttmann-Schweiger N, Fiebig J, Poethko-Muller C, Prutz F, Sarganas G, et al. Seroprevalence of SARS-CoV-2 among children and adolescents in Germany-an overview. Bundesgesundheitsblatt - Gesundheitsforsch Gesundheitsschutz 2021;64:1483-91.

24. Hippich M, Sifft P, Zapardiel-Gonzalo J, Bohmer MM, Lampasona $\mathrm{V}$, Bonifacio $\mathrm{E}$, et al. A public health antibody screening indicates a marked increase of SARS-CoV-2 exposure rate in children during the second wave. Med 2021;2:571-2.

25. Fagni F, Simon D, Tascilar K, Schoenau V, Sticherling M, Neurath MF, et al. COVID-19 and immune-mediated inflammatory diseases: effect of disease and treatment on COVID-19 outcomes and vaccine responses. Lancet Rheumatol 2021;3: e724-36.

26. Long QX, Tang XJ, Shi QL, Li Q, Deng HJ, Yuan J, et al. Clinical and immunological assessment of asymptomatic SARS-CoV-2 infections. Nat Med 2020;26:1200-4.

27. Weisberg SP, Connors TJ, Zhu Y, Baldwin MR, Lin WH, Wontakal S, et al. Distinct antibody responses to SARS-CoV-2 in children and adults across the COVID-19 clinical spectrum. Nat Immunol 2021; 22:25-31.

28. Loske J, Rohmel J, Lukassen S, Stricker S, Magalhaes VG, Liebig J, et al. Pre-activated antiviral innate immunity in the upper airways controls early SARS-CoV-2 infection in children. Nat Biotechnol 2021. https://doi.org/10.1038/s41587-02101037-9.

29. Abela IA, Pasin C, Schwarzmuller M, Epp S, Sickmann ME, Schanz $M M$, et al. Multifactorial seroprofiling dissects the contribution of pre-existing human coronaviruses responses to SARS-CoV-2 immunity. Nat Commun 2021;12:6703.

30. Dowell AC, Butler MS, Jinks E, Tut G, Lancaster T, Sylla P, et al. Children develop robust and sustained cross-reactive spikespecific immune responses to SARS-CoV-2 infection. Nat Immunol 2022;23:40-9.

31. Grandjean L, Saso A, Torres A, Lam T, Hatcher J, Thistlethwayte R, et al. Humoral response dynamics following infection with SARSCoV-2. Clin Infect Dis 2021;2020:ciab607.

32. Laub O, Leipold G, Toncheva AA, Peterhoff D, Einhauser S, Neckermann P, et al. Symptoms, SARS-CoV-2 antibodies, and neutralization capacity in a cross sectional-population of German children. Front Pediatr 2021;9:678937. 
33. Bohn MK, Hall A, Wilson S, Taher J, Sepiashvili L, Adeli K. Pediatric evaluation of clinical specificity and sensitivity of SARS-CoV-2 IgG and IgM serology assays. Clin Chem Lab Med 2021;59:e235-7.

34. Hippich M, Holthaus L, Assfalg R, Zapardiel-Gonzalo J, Kapfelsperger $\mathrm{H}$, Heigermoser $\mathrm{M}$, et al. A public health antibody screening indicates a 6-fold higher SARS-CoV-2 exposure rate than reported cases in children. Med 2021;2:149-63.

35. Robert-Koch-Institute. Epidemiol Bull 2021;37:1-18.
36. Bhuiyan MU, Stiboy E, Hassan MZ, Chan M, Islam MS, Haider N, et al. Epidemiology of COVID-19 infection in young children under five years: a systematic review and meta-analysis. Vaccine 2021; 39:667-77.

Supplementary Material: The online version of this article offers supplementary material (https://doi.org/10.1515/cclm-2022-0037). 